\title{
STEP BY STEP MAGNETIC RESONANCE IMAGING IN A CASE OF CARDIAC AMYLOIDOSIS - IT IS NOT ALL ABOUT AMYLOID DEPOSITION!
}

\author{
Sebastian Onciul1 ${ }^{1,2}$, Ioana Petre ${ }^{1,2}$, Radu Nicolaescu ${ }^{1,3}$, Andrei Voicovici $^{3}$, Răzvan Capșa $^{1,3}$ \\ ${ }^{1}$ Carol Davila University of Medicine and Pharmacy, Bucharest \\ ${ }^{2}$ Clinical Emergency Hospital Bucharest \\ ${ }^{3}$ Fundeni Clinical Institute, Bucharest \\ Corresponding author: Sebastian Onciul \\ e-mail: sebastian.onciul@gmail.com
}

Keywords: cardiac amyloidosis, magnetic resonance, left ventricular hypertrophy.

It is often said that cardiac magnetic resonance (CMR) has the diagnostic power of a noninvasive myocardial biopsy. We illustrate this concept by stepwise analysis of a CMR exam in 65-year-old patient referred to our department for tissue characterization of a left ventricular (LV) hypertrophy of unknown etiology. The subsequent complete laboratory workup has diagnosed cardiac amyloidosis (CA) of AL type (light chain CA). The reader will become acquainted with the common sequences of the clinical CMR protocol in CA.

The conventional T2-weighted imaging is a qualitative method which can detect only focal oedema as an area of hyperintensity (oedema) surrounded by lower intensity (normal) myocardium. In image $\mathbf{C}$. homogenous intensity of the myocardium is seen, excluding focal oedema, however, diffuse myocardial oedema (such is the case of CA) cannot be excluded. T2 mapping (D) is a quantitative technique allowing the measurement of T2 value in each voxel. A T2 value higher than $50 \mathrm{~ms}$ is diagnostic for myocardial oedema (at 1.5 Tesla). In our case, a T2 value of $65 \mathrm{~ms}$ was highly suggestive of myocardial oedema accompanying the amyloid deposition(1). Short axis quantitative T1 maps before (E) and after (F) Gadolinium-based contrast agent injection. Amyloidosis is characterized by the highest native T1 times among all heart conditions, with values $>1164 \mathrm{~ms}$ being highly suggestive of CA (2). Introducing native and post-contrast $\mathrm{T} 1$ values in a formula, the extracellular volume fraction (ECV) can be calculated. In CA the interstitium is expanded due to amyloid deposition and thus ECV can accurately and non-invasively estimate the myocardial amyloid burden. In our patient the calculated ECV was $76 \%$ (normal <30\%) ${ }^{(3)}$. 


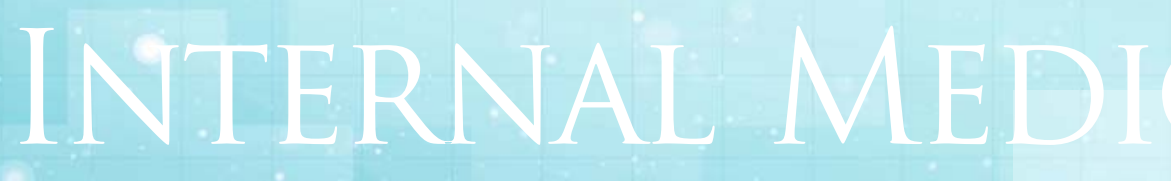

\section{Images in Medicine}

Two chambers views acquired early (G) and 10 minutes after (H) Gadolinium injection. The early Gadolinium enhancement (EGE) images are acquired in the first minute after contrast injection, allowing for detection of poorly perfused tissues such as areas of microvascular obstruction (MVO). In our case, MVO was identified as a dark subendocardial rim (yellow arrows) surrounded by normally enhanced myocardium.

On the other hand, late Gadolinium enhancement (LGE) images are usually pathognomonic for CA: the myocardium appears bright with a dark blood pool appearance (Gadolinium is an extracellular agent which concentrates in tissues with enlarged extracellular spaces such as scars or amyloid infiltration). In the case of our patient, it can be seen that Gadolinium is retained in areas with the highest amyloid burden, mainly the basal LV segments with relative apical sparing (H., yellow arrows).

By gathering information from all the sequences of the CMR protocol we were able to demonstrate that CA is not characterized solely by amyloid deposition in the interstitium. On the contrary, several other

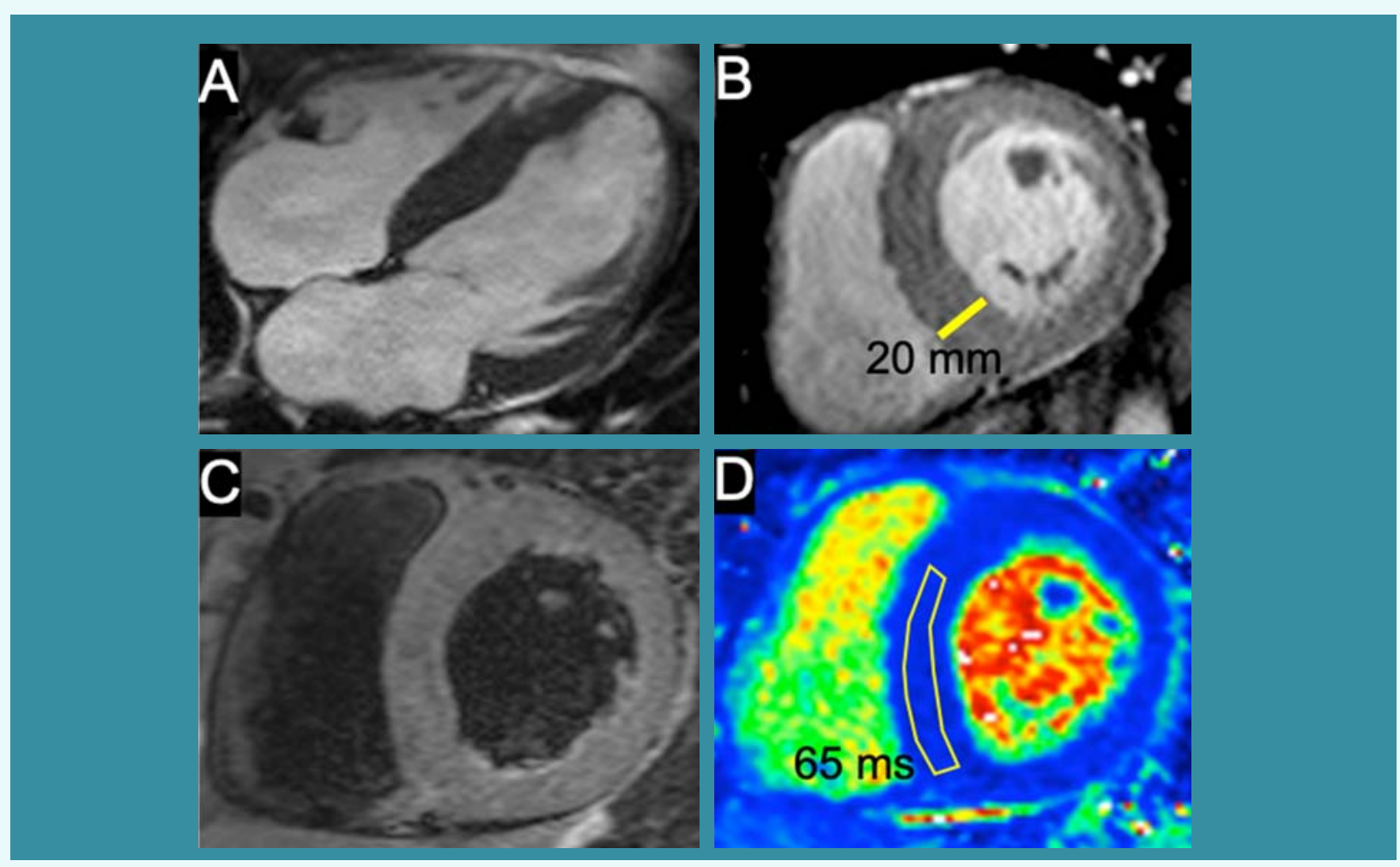



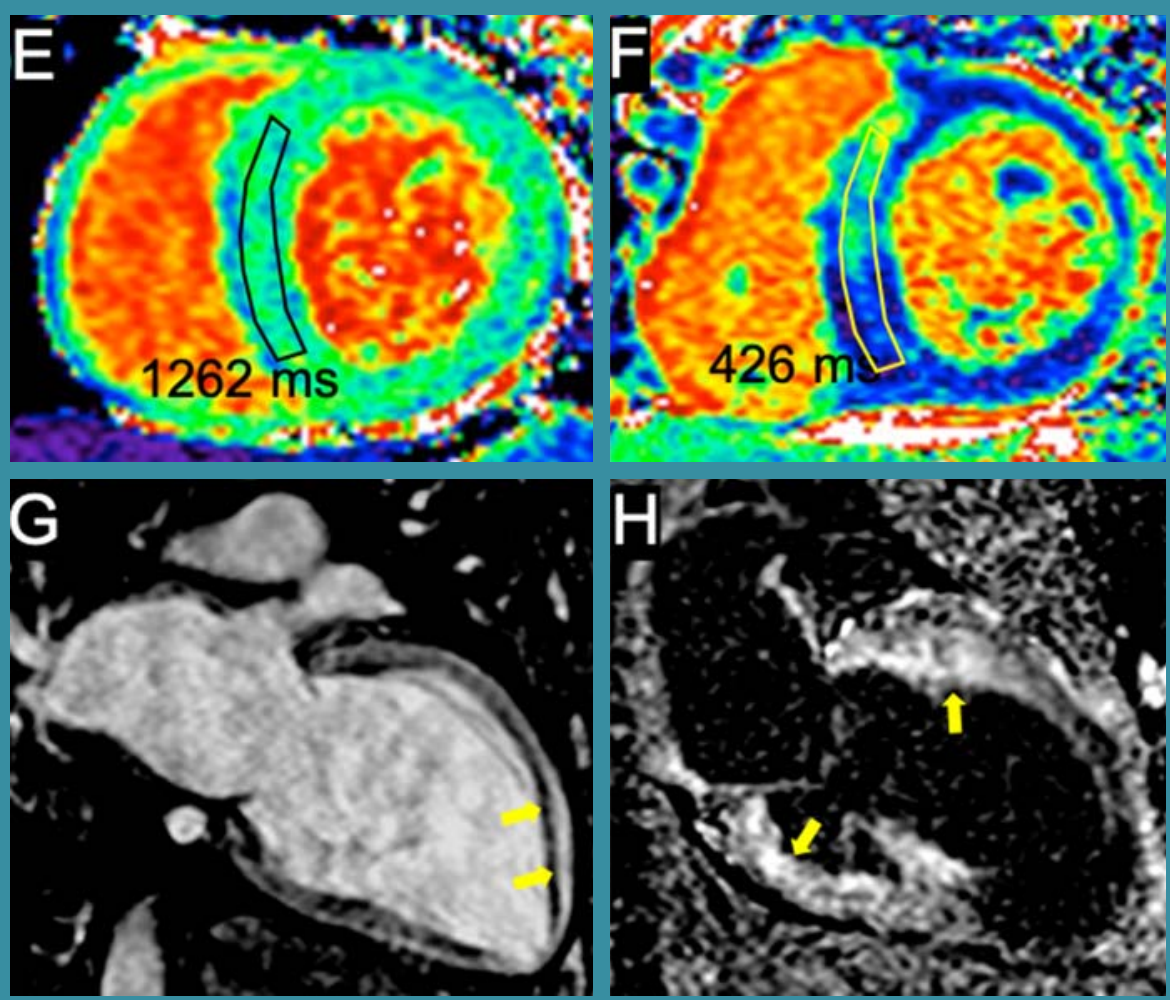

Figure 1. Non-contrast cine images (balanced steady-state free precession) in 4 chambers (A) and short axis (B) views respectively allow for morphological and functional assessment. The left ventricle (LV) was dilated and the interventricular septum was severely and asymmetrically hypertrophied. The LV ejection fraction was 35\%. The right ventricular (RV) free wall was not thickened, however RV was severely dysfunctional, with an ejection fraction of $25 \%$. The atria were only mildly dilated.

Imaging of myocardial oedema in short axis view using the conventional T2-weighted sequences (C) and the more recently developed T2 mapping technique (D).

pathophysiological processes occur in AL CA, such as: myocardial oedema, myocardiocyte necrosis and microvascular obstruction. These phenomena express the central role of direct light chain toxicity on myocardiocytes resulting in a toxic myocarditis. In AL CA the direct myocardial toxicity of the light chains plays a more important role in the pathophysiology of the disease than strictly interstitial deposition of amyloid.

CMR is the only non-invasive imaging modality able to simultaneously quantify the amyloid burden as well as to identify myocardial oedema, necrosis and microvascular obstruction, providing important prognostic information. This is why CMR undoubtedly deserves the designation of noninvasive myocardial biopsy and should be offered to all CA patients.

\section{References}

1. Kotecha T, Martinez-Naharro A, Treibel TA, Francis $R$, Nordin S, Abdel-Gadir A, et al. Myocardial Edema and Prognosis in Amyloidosis. J Am Coll Cardiol [Internet]. 2018 Jun;71(25): 291931. Available from: https:// linkinghub.elsevier.com/retrieve/pii/S0735109718 345716 2. Baggiano A, Boldrini M, Martinez-Naharro A, Kotecha T, Petrie $A$, Rezk $T$, et al. Noncontrast Magnetic Resonance for theDiagnosis of Cardiac Amyloidosis. JACC Cardiovasc Imaging [Internet]. 2019 Jun 12;3055. Available from: http://imaging.onlinejacc.org/ content/ early/ 2019/ 06/07/j.jcmg.2019.03.026.abstract

3. Martinez-Naharro A, Kotecha T, Norrington K, Boldrini M, Rezk T, Quarta C, et al. Native T1 and Extracellular Volume inTransthyretin Amyloidosis. JACC Cardiovasc Imaging [Internet]. 2019 May 1;12(5):810 LP 819. Available from: http://imaging.onlinejacc.org/content/12/5/810.abstract 